\title{
Atypische Beschäftigung und der staatliche Schutz vor Altersarmut - ein europäischer Vergleich
}

KARIN SCHULZE

BUSCHOFF $^{1}$

Dr. Karin Schulze Buschoff ist Privatdozentin für komparative Arbeits- und Sozialpolitik an der FU Berlin.

In vielen Ländern bilden die atypisch Beschäftigten mit einem Anteil von jeweils deutlich über einem Drittel an allen Beschäftigten einen beachtlichen Teil der Gesamtbeschäftigten. Das deutsche System der staatlichen Altersvorsorge ist aufgrund der starken Lohnzentrierung und der Orientierung am Äquivalenzprinzip nur unzureichend auf atypische Beschäftigungsverhältnisse und weitere Flexibilisierungstendenzen eingestellt. Fraglich bleibt, ob ein Festhalten an der traditionellen deutschen Rentenpolitik den durch zunehmend diskontinuierlicher Erwerbsverläufe entstehenden Herausforderungen gerecht werden kann. Als zukunftsfähiger und überzeugender in Hinblick auf die Bewältigung dieser Herausforderungen stellen sich Grundrentensysteme dar, z. B. die Systeme in Dänemark oder in den Niederlanden.

\section{Einleitung}

Die nationalen Arbeitsmärkte der EUMitgliedstaaten stehen vor einen hohen Anpassungsdruck. Globalisierung, verschärfter Wettbewerb der auf internationalen Märkten agierenden Unternehmen, technologischer Wandel, demographische Veränderungen und steigende Frauenerwerbstätigkeit, Flexibilisierungsanforderungen durch neue Organisationsformen und ein sektoraler Wandel hin zum Dienstleistungssektor führen als Bestandteile dieses Anpassungsdrucks bereits seit den 1970er Jahren zu einem grundlegenden strukturellen Wandel der Arbeitsmärkte.

Dieser strukturelle Wandel der Arbeitsmärkte geht einher mit einem sozialen Wandel in Form einer stärkeren Individualisierung der Erwerbsverläufe. Erwerbsverläufe werden insgesamt »brüchiger«. Übergänge zwischen Er- werbsformen und Nicht-Beschäftigung werden häufig Bestandteil von diskontinuierlichen Erwerbsverläufen (Schmid 2000). Flexibilitätsanforderungen entsprechend ist der Anteil der sogenannten »atypischen « d. h. vom Normalarbeitsverhältnis abweichenden Arbeitsverhältnisse, also befristete Beschäftigungen, Solo-Selbstständigkeit, geringfügige Beschäftigungen und Teilzeitbeschäftigungen, in den letzten Jahren in der Mehrzahl der europäischen Länder gestiegen (Schulze Buschoff/Protsch 2008; Schmid/ Protsch 2009; Schmid 2010).

Vor dem Hintergrund zunehmender Flexibilisierung hat die EU-Kommission in den letzten Jahren eine umfassende Debatte über den Begriff der Flexicurity angestoßen. Mit dem Begriff der Flexi-

\footnotetext{
1 Unter Mitarbeit von Jutta Höhne, Forschungsassistentin am Wissenschaftszentrum Berlin für Sozialforschung (WZB)
} 


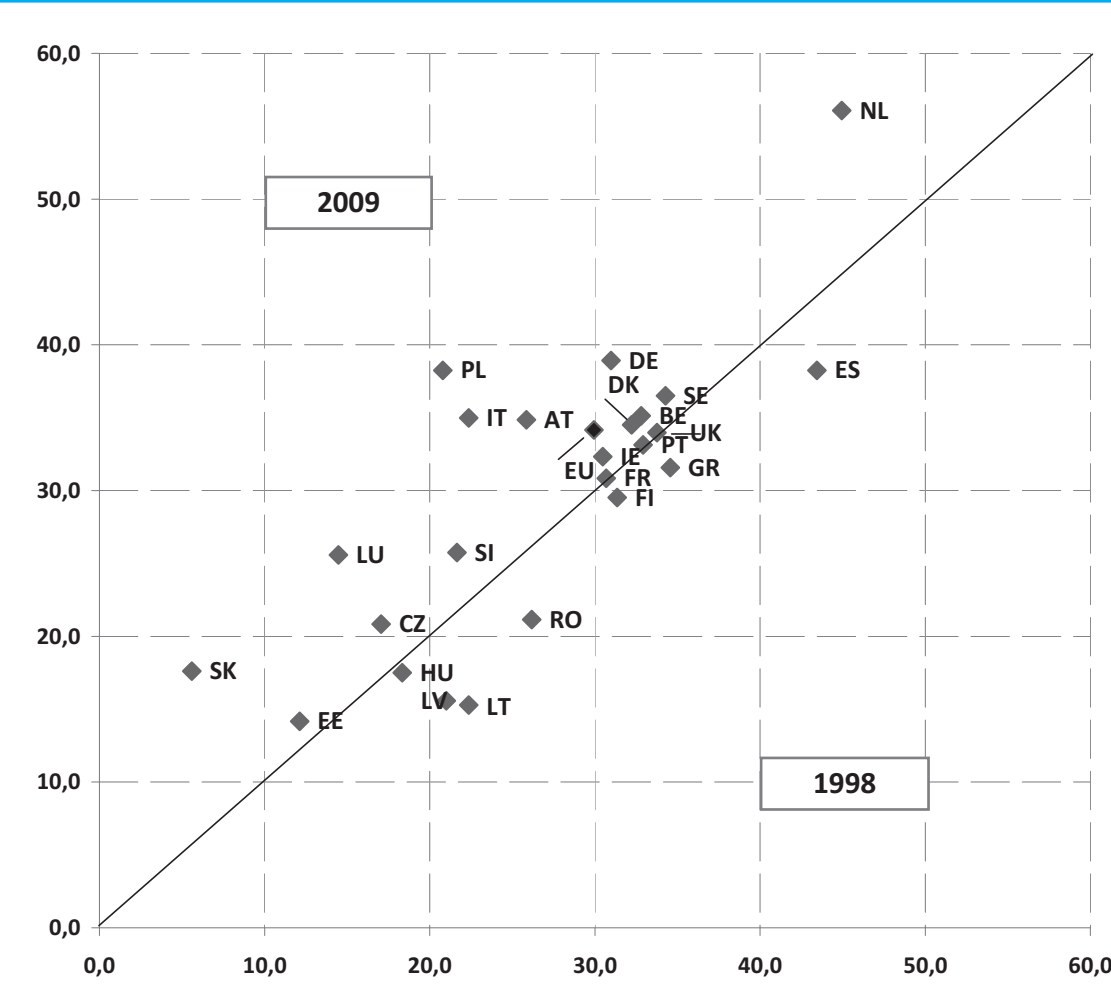

Abbildung 1: Anteil atypisch Erwerbstätiger an allen Erwerbstätigen (Alter 15-64), in Prozent, 1998 und 2009

Quelle: EUROSTAT, Labour Force Survey, eigene Berechnungen, Anmerkung: EU 2009 einschließlich BG, CY, MT

curity ist die Verbindung zwischen einer größeren Flexibilität (flexibility) und einem hierauf abgestimmten Sozialschutz (security) gemeint. Der Begriff für diese Verbindung wurde im Kontext der niederländischen Reformen der 1990er Jahre geprägt. Nach dem Konzept der »flexicurity « wird die Verbindung zwischen Flexibilität und Sicherheit nicht als Paradox, sondern im Gegenteil als Voraussetzung für die Akzeptanz für Flexibilität betrachtet (Leschke et al. 2006: 1). "Flexicurity" gilt als PolicyStrategie, die die Flexibilität des Arbeitsmarktes und die soziale Sicherheit - und zwar vor allem die der schwächeren Gruppen - zu stärken sucht (Wilthagen 1998; Wilthagen/Tros 2004: 169).

Seit einigen Jahren fließt das Konzept der Flexicurity in die Europäische Beschäftigungsstrategie ein. Die jährlichen Europäischen Beschäftigungsberichte stellen schon seit 2002 ausführlich verschiedene Formen von FlexicurityArrangements dar. Im März 2008 hat der Europäische Rat den neuen Zyklus der integrierten Leitlinien der Lissabon-Strategie gebilligt, unter Berücksichtigung gemeinsamer Grundsätze der Flexicurity. Die Kommission hat, ebenfalls im Jahr 2008, auf Aufforderung des Rates eine öffentliche Initiative in Form einer Flexicurity Mission gestartet. Die Initiative zielt darauf ab, den Mitgliedstaaten bei der Integration der gemeinsamen Grundsätze der Flexicurity zu helfen (Rat der Europäischen Union 2008). Flexicurity gilt in der Europäischen Union als das derzeit gefragteste Konzept für die Reformen der Arbeitsmärkte, der Arbeitsgesetzgebung und der Beschäftigungspolitik (Bredgaard et al. 2009: 31).

Im Mittelpunkt dieses Beitrags steht die Frage, ob die staatlichen sozialen Sicherungssysteme der EU-Mitgliedstaaten besonders mit Blick auf die Altersvorsorge der atypisch Beschäftigten der Flexicurity-Strategie gerecht werden. Können die staatlichen Altersrentensysteme den atypisch Beschäftigten einen ausreichenden Schutz vor Altersarmut gewährleisten? Bevor die verschiedenen nationalen Systeme der sozialen Absicherung atypisch Beschäftigter in den Blick genommen werden, soll zunächst eine empirische Analyse einen kurzen Überblick über den Wandel der Arbeitsverhältnisse vermitteln.

\section{Wandel der Arbeitsverhältnisse in Europa}

In vielen Ländern der EU 27 ist der Anteil atypisch Beschäftigter an der $\mathrm{Ge}$ samtzahl der Beschäftigten recht ausgeprägt. In den meisten Ländern zeigt sich zudem eine Zunahme der atypisch Beschäftigten im Zeitraum 1998 bis 2009 (der gleiche Trend zeigt sich, wenn der Prozentanteil der atypisch Beschäftigten an der Gesamtbevölkerung betrachtet wird (Schmid/ Protsch 2009)).

Dieser Abbildung liegt ein zusammenfassender Indikator zugrunde, der die Mehrfachzählung von Personen, auf die mehrere Merkmale atypischer Beschäftigung zutreffen, ausschließt. Dafür werden atypisch Beschäftigte wie folgt definiert:

- Beschäftigte mit befristeten Verträgen

- In Vollzeit arbeitende Solo- Selbstständige

- Teilzeitbeschäftigte mit Festanstellung und in Teilzeit arbeitende Solo-Selbstständige (Arbeitszeit $>15$ Stunden/Woche), die sich selbst als in Teilzeit arbeitend definieren

- Geringfügig Beschäftigte mit Festanstellung oder geringfügig beschäftigte Solo-Selbstständige (Arbeitszeit $\leq 15$ Stunden/Woche).

In den meisten Ländern bilden die atypisch Beschäftigten mit einem Anteil von jeweils über einem Drittel (bzw. in den Niederlanden sogar der Hälfte) an allen Beschäftigten einen beachtlichen Teil der Gesamtbeschäftigten. Der Anteil einzelner Formen atypischer Beschäftigung ist in einigen Ländern so hoch, dass es eigentlich nicht gerechtfertigt scheint, von atypischer Beschäftigung zu sprechen. So zählen die Teilzeitbeschäftigung in den Niederlanden, die Solo-Selbstständigkeit in Italien und die befristete Beschäftigung in Polen aufgrund ihrer hohen Anteile wohl eher zu den »typischen " Erwerbsformen; der Vergleichbarkeit halber wird jedoch die Bezeichnung "atypische Beschäftigung " beibehalten. Frauen, gering Qualifizierte und jüngere Personen unter 30 Jahren sind besonders häufig atypisch beschäftigt. Summa summarum hat der Anteil atypischer Beschäftigung seit Ende der 1990er Jahre zugenommen. Es bestehen jedoch deutliche Unterschiede in den 
Anteilen und der Entwicklung einzelner Formen der atypischen Beschäftigung zwischen den einzelnen Ländern.

\section{Die staatliche Alterssicherung der atypisch Beschäftigten im Ländervergleich}

Im Folgenden werden die staatlichen Alterssicherungsysteme skizziert und der Frage nachgegangen, ob und inwiefern sie auf die atypischen Beschäftigungsformen eingestellt sind. Für einen exemplarischen Ländervergleich werden Deutschland, das Vereinigte Königreich, die Niederlande, Italien, Dänemark und Polen ausgewählt. Damit wird ein breites Spektrum von wirtschaftlichen Schwerpunkten, institutionellen und sozialpolitischen Rahmenbedingungen sowie kulturellen Voraussetzungen innerhalb der Vielfalt Europas abgedeckt.

\section{Dänemark}

Die Grundpfeiler des gesetzlichen Rentensystems in Dänemark sind das Volksrentensystem und das Arbeitsmarkt-Zusatzrentensystem. Eine steuerfinanzierte Volks- bzw. Altersgrundrente bietet armutsvermeidende Einkommenssicherheit für alle Einwohner, die ihren Wohnsitz ausreichend lange in Dänemark hatten. Die Mitgliedschaft hängt allein vom Wohnen in Dänemark bzw. der Einwohnerschaft $\mathrm{ab}$ und nicht davon, ob der/die Versicherte eine Beschäftigung ausgeübt hat. Das Volksrentensystem wird durch ein einkommensbezogenes, jedoch relativ schwach am Äquivalenzprinzip orientiertes ArbeitsmarktZusatzrentensystem (ATP) ergänzt. Da die Dauer der Versicherung in die Berechnung mit einfließt, können Nachteile für lückenhafte Erwerbsbiografien entstehen. Das Zusatzrentensystem ATP schließt geringfügig Beschäftigte mit einer Arbeitszeit unter neun Stunden und Solo-Selbstständige ohne vorheriges Beschäftigungsverhältnis aus. SoloSelbstständige sind wegen fehlender Betriebszugehörigkeit auch bezüglich der betrieblichen Alterssicherung im Nachteil. Diese erreicht bei den abhängig Beschäftigten einen Deckungsgrad von $90 \%$.

Anspruch auf die Volksrente erwirbt jeder Einwohner, der ab dem 15. Lebensjahr mindestens drei Jahre in Dänemark gewohnt hat. Die Volksrente wird ab dem 65. Lebensjahr ausgezahlt und setzt sich aus einem Grundbetrag und einer Rentenzulage zusammen. Es handelt sich um gesetzlich festgelegte Pauschalbeträge. Die volle Rente erhält man, wenn man zwischen dem 15. und 65. Lebensjahr mindestens 40 Jahre in Dänemark gewohnt hat. Die volle Rente setzt sich dann aus folgenden Beträgen zusammen: der Grundbetrag betrug im Jahr 2006 DKK 4648 im Monat für eine alleinstehende Person, das entspricht etwa 623 Euro. Die Rentenzulage für eine alleinstehende Person betrug 2006 DKK 4679 pro Monat, das entspricht etwa 628 Euro (OECD 2007). Alle in voller Höhe ausgezahlten staatlichen Zahlungen aus dem steuerfinanzierten Altersgrundrentensystem setzen eine 40jährige Einwohnerschaft voraus. Kürzere Phasen der Einwohnerschaft bedeuten anteilig entsprechend geringere Auszahlungssummen (Kürzungen pro fehlendes Jahr um 1/40tel). Bezieht der Rentenempfänger bzw. die Rentenempfängerin oder ggfs. der Ehepartner weitere Leistungen bzw. Einkommen neben der Rente, so kann die Rente gekürzt werden oder ganz wegfallen. Die Volksrente wird monatlich gezahlt und jährlich an die Lohnentwicklung angepasst (OECD 2007).

\section{Deutschland}

In Deutschland gibt es keine Altersgrundrente, aber eine bedürftigkeitsgeprüfte Mindestsicherung im Alter (außerhalb des Rentensystems (Sozialhilfe)). Wie das Arbeitslosenversicherungssystem ist auch das erwerbsbezogene Alterssicherungsregelsystem stark am Äquivalenzsystem orientiert, d. h. für die Höhe der Leistungen ist die Erwerbsbiografie bzw. die Dauer der Beschäftigungszeiten und die Höhe der Einkommen von entscheidender Bedeutung. Von dem staatlichen System der Alterssicherung sind ein Großteil der Selbstständigen und die geringfügig Beschäftigten (400 Euro-Jobs bzw. Minijobber/innen) ausgeschlossen.

Auch wenn rund ein Drittel der Gesamtausgaben der Deutschen Rentenversicherung durch Bundeszuschüsse bestritten werden, um etwa anrechenbare aber beitragsfreie Zeiten, z. B. der Kindererziehung, auszugleichen, gilt deutlich das Prinzip: die Rente spiegelt die Erwerbsbiografie wider. Die bestimmenden Faktoren der Leistungen sind in erster Linie die Höhe der während des gesamten Versicherungslebens durch Beiträge versicherten Arbeitsentgelte.

Die politische Stoßrichtung, zu Lasten der Leistungen in erster Linie den Beitragssatz zu stabilisieren und die »Rentenkürzungsreformen " der letzten Jahre z. B. in Form der Verschlechterung der rentenrechtlichen Berücksichtigung von Ausbildungszeiten oder von Zeiten des Bezugs von Arbeitslosengeld II führen im Zusammenhang mit der Zunahme atypischer Beschäftigung, der Ausweitung des Niedriglohnsektors und zunehmend diskontinuierlicher Erwerbsverläufe auf längere Sicht zu einer Erhöhung des Armutsrisikos für Rentner und Rentnerinnen. Schon heute zeigt sich, dass die Rentenzahlbeträge deutlich sinken. Ein Vergleich der Renten des Rentenbestands, also der derjenigen Personen, die im Jahr 2008 bereits Rentner und Rentnerinnen waren und des Rentenzugangs, also derjenigen, die 2008 Rentner und Rentnerinnen wurden, zeigt deutlich die Absenkung der Nettozahlbeträge. Während die Männer unter den Bestandsrentnern in den alten Bundesländern noch eine durchschnittliche Nettorente von 950 Euro bezogen, erhielten die Zugangsrentner nur einen Betrag von 822 Euro. Die Renten der Frauen sinken zwar nicht so schnell wie die der Männer, sie bewegen sich aber bereits heute auf einem Armutsgefährdungsniveau. Die Bestandsrentnerinnen aus den alten Bundesländern erhielten 2008 monatlich 485 Euro netto, die Zugangsrenterinnen nur 468 Euro (Kerschbaumer 2010a, Berechnungen auf der Grundlage von Zahlen der Rentenversi-

\section{In Bezug auf die soziale} Sicherung von selbständig Erwerbstätigen stellt Deutschland im europäischen Vergleich eine
Besonderheit dar.

cherung Bund, Stand 31.12.2008, nicht erfasst werden Ansprüche in anderen Systemen außerhalb der GRV).

Hinzu kommt, dass Frauen im Vergleich zu Männern seltener von betrieblicher Altersvorsorge profitieren: 
Während rund ein Drittel der Männer Anwartschaften aus einer betrieblichen Altersvorsorge haben, sind es nur ca. 7\% der Frauen. Dabei betragen die Rentenbeträge der Frauen mit durchschnittlich 213 Euro weniger als die Hälfte als die der Männer (Kerschbaumer 2010a: 8).

In Bezug auf die soziale Sicherung von selbständig Erwerbstätigen stellt Deutschland im europäischen Vergleich eine Besonderheit dar: Während in der Mehrzahl der europäischen Länder die Selbstständigen durch die staatlichen Pflichtversicherungssysteme systematisch erfasst werden, ist die Pflichtversicherung in Deutschland entsprechend der Tradition der Bismarckschen Sozialversicherung auf wenige Sondergruppen Selbstständiger (bzw. Scheinselbstständiger) begrenzt. Dahinter steht die Vorstellung, dass die Selbstständigen für sich selbst vorsorgen können und nicht des kollektiven Schutzes der Solidargemeinschaft der Versicherten bedürfen. Dies gilt, mit Ausnahme von Sonderregelungen, die einzelne Gruppen von Selbstständigen in die Sozialversicherung mit einbeziehen, auch heute noch. ${ }^{2}$

Offensichtlich ist, dass auch weitere Gruppen von Selbstständigen, insbesondere unter den »neuen Selbstständigen ", nicht anders als die bislang schon pflichtversicherten Gruppen von Selbstständigen und die abhängig Beschäftigten auf den Verkauf ihrer Arbeitskraft angewiesen und den sozialen Risiken Alter, Krankheit und Arbeitsbzw. Auftragslosigkeit in gleicher Weise ausgesetzt sind. Vielfach gefordert wird deshalb die Erweiterung der staatlichen Pflichtversicherung der Altersvorsorge um Selbstständige aller Berufsgruppen.

\section{Italien}

In Italien gibt es kein Grundsicherungssystem für die Altersrente, verschiedene berufsgruppenspezifische Alterssicherungsregelsysteme (sog. Fonds) sind jedoch für alle Erwerbstätigen (einschließlich der Selbstständigen) obligatorisch. Der FLDP (Fondo pensioni lavoratori dipendenti) als der größte Fond ist versicherungsbasiert und wird vorwiegend über Beiträge im Wege des Umlageverfahrens finanziert. Die Rentenhöhe hängt bezüglich des Zeit- und Einkommensfaktors von der vorherigen Erwerbsbiografie ab. Da sich der
Aufbau, die Leistungen und die Finanzierung der verschiedenen Fonds zum Teil erheblich unterscheiden, kann ein beruflicher Wechsel bzw. ein Wechsel von einem Fond zum anderen, mit Nachteilen verbunden sein (Schulze Buschoff 2004: 7).

\section{Im Jahr 2010 wurde für das Rentensystem mehr als ein Drittel aller laufenden Ausgaben der öffentlichen Haushalte aufgebracht.}

In den 1990ern wurde das Altersrentensystem in Italien umfassend reformiert. Hintergrund war der erhebliche Kostendruck durch großzügige Regelungen. So hatten Staatsbedienstete bereits nach 15 oder 20 Beitragsjahren Anspruch auf Rentenzahlungen. Abhängig Beschäftigte konnten im Alter von Anfang 50 mit rund 70 Prozent ihres letzten Gehalts in den Ruhestand gehen. Für alle wurden die Renten nach den letzten Gehältern berechnet, mit 2 Prozent des Gehalts je Beitragsjahr, was effektive Renten von 90 Prozent des letzten Nettogehalts hervorbrachte. Die Rentenkosten wurden deshalb für das Jahr 2040 auf mehr als 23 Prozent des BIP geschätzt (FAZ 2011).

Zum 1. Januar 1996 wurde als bedeutendster Teil der Reform Dini die Änderung der Berechnungsmethode der Rentenhöhe von einem verdienstorientierten System - »sistema retributivo « - auf das beitragsorientierte »sistema contributivo" eingeführt. Nach dem alten System wird nur das Einkommen der letzten fünf Jahre betrachtet und als Ausgangspunkt für die Ermittlung der Rentenhöhe verwendet. Im sistema contributivo ist hingegen die gesamte Erwerbsbiographie für die spätere Rentenhöhe erheblich; daneben bemisst sich die spätere Rentenhöhe nicht nach dem Einkommen, sondern nach der Summe der während des Erwerbslebens geleisteten Beiträge.Insgesamt gilt trotz der erfolgten Reformen noch immer, dass das italienische Rentensystem extrem teuer ist. Im Jahr 2010 wurde für das Rentensystem mehr als ein Drittel aller laufenden Ausgaben der öffentlichen Haushalte aufgebracht. Die italienischen Sozialbeiträge, 32,7 Prozent für den Arbeitgeber und 9,19 Prozent für den Arbeitnehmer, sind im internationalen Vergleich hoch und müssen fast ausschließlich für die Renten verwendet werden, um das System stabil zu halten (FAZ 2011).

In Italien hat die Selbständigkeit traditionell eine hohe Bedeutung, der Anteil der Selbstständigen an allen Erwerbstätigen ist im Ländervergleich einer der höchsten in ganz Europa. In den letzten Jahren ist in Italien eine intensive Debatte um den rechtlichen Schutz für Selbständige und vor allem für wirtschaftlich abhängige Selbständige geführt worden.

Dies hat zur Folge, dass alle Gruppen von Selbständigen bzw. von Personen im Grenzbereich zwischen selbständiger und abhängiger Erwerbsarbeit inzwischen in die obligatorischen Systeme der sozialen Sicherung integriert sind. Mit Ausnahme der Arbeitslosigkeit werden soziale Risiken durch die staatlichen Systeme abgedeckt: so sind alle Gruppen von Selbständigen in das Gesundheitssystem integriert, haben Ansprüche im Falle von Mutterschaft und generieren Ansprüche auf Rentenzahlungen. Von Nachteil ist jedoch, dass die Regelungen selektiv und partikularistisch sind, d. h. je nach Zugehörigkeit zu einem bestimmten Rentenfond erhebliche Unterschiede aufweisen können.

\section{Niederlande}

Mit dem Algemene Ouderdomswet (AOW = Gesetzliche Altersrente) wurde in den Niederlanden 1957 ein umfassendes staatliches Grundversorgungssystem

2 Bei diesen einzelnen Gruppen von Selbstständigen wurde davon ausgegangen, dass die Annahme fehlender Schutzbedürftigkeit nicht gerechtfertigt ist und sie wurden schrittweise in die staatliche Alterssicherung integriert. So bestehen heute für etwa ein Viertel der Selbstständigen obligatorische Sondersysteme, wobei die Bedingungen je nach Berufsgruppe sehr unterschiedlich sind. Obligatorische Alterssicherungssysteme gelten für Hausgewerbetreibende, Lehrer/innen, Erzieher/innen, Pflegepersonal, Hebammen, Seelots/inn/en, Küstenschiffer/innen und Küstenfischer/ innen; Handwerker/innen mit Eintrag in die Handwerksrolle und Bezirksschornsteinfegermeister/innen; Künstler/innen und Publizist/inn/en; Landwirt/inn/e/n; sowie Freie Berufe wie Rechtsanwält/inn/e/n, Notar/inn/e/n oder Ärzt/inn/e/n. Für selbstständige Künstler/innen und Publizist/inn/ en besteht die Versicherungspflicht in der in der Kranken- und Rentenversicherung mit der Schaffung der Künstlersozialkasse (KSK) seit 1983. 
eingeführt. Das AOW basiert auf einer obligatorischen Alterssicherung nach dem Umlageverfahren für sämtliche in den Niederlanden wohnhaften Personen. Die AOW-Grundsicherung ist eine Pauschalleistung, die nicht von den Beiträgen abhängig ist, die während des Erwerbslebens eingezahlt wurden.

Der niederländische Staat stellt allen Bürgern ab dem 65. Lebensjahr eine

\section{Die Niederlande stellt allen Bürgern ab dem 65. Lebensjahr eine staatliche Grundsicherung zur Verfügung.}

staatliche Grundsicherung zur Verfügung, die nur auf die Einwohnerschaft, d. h. auf das Wohnen in den Niederlanden abgestellt ist und bei der die Erwerbstätigkeit keine Rolle spielt. Auch Einwohner, die nie einer versicherungspflichtigen Tätigkeit nachgegangen sind und geringfügig Beschäftigte haben Anspruch auf eine staatliche Rente. Es gibt keine Bedürftigkeitsprüfung. Weder ein vorgezogener Rentenbezug noch ein Ausstieg aus dem staatlichen Pflichtversicherungssystem sind möglich.

Die maximale Höhe dieser Grundrente ist für jeden Empfänger gleich und richtet sich nach dem gesetzlichen Nettomindestlohn. Für jedes AOW-Versicherungsjahr werden $2 \%$ des vollen Betrages der AOW-Grundrente aufgebaut. Personen, die zwischen der Vollendung ihres 15. und 65. Lebensjahres nicht ununterbrochen nach dem AOW versichert gewesen sind, d. h. nicht wohnhaft in den Niederlanden waren, haben keinen Anspruch auf eine volle AOW. Der volle Betrag der AOW wird dann für jedes fehlende Versicherungsjahr um $2 \%$ gekürzt (Niederlandeweb 2011).

Die staatliche Grundrente wird durch steuerähnliche Beiträge finanziert, die auf das Einkommen erhoben werden. Jede Person in den Niederlanden mit einem Einkommen ist verpflichtet, diese Beiträge zu zahlen. Beiträge werden unter anderem erhoben auf Einkünfte aus unselbstständiger Tätigkeit, Renten, Sozialleistungen, Einkünfte aus Gewerbebetrieben oder selbstständiger Arbeit und Mieten (Bieber 2003: 137).

Der Beitragssatz für die gesetzliche Rentenversicherung (AOW) beträgt 17,9 Prozent, für die Hinterbliebenenversor- gung (ANW) 1,25 Prozent. Die Beiträge werden allein von den Arbeitnehmern entrichtet, Arbeitgeber müssen keine Beiträge zahlen. Das Einkommen wird höchstens bis zu 28.850 Euro pro Jahr mit Beiträgen zur Volksversicherung belastet. Die Beiträge für die Invaliditätsversicherung werden dagegen allein vom Arbeitgeber entrichtet. Die Höhe der ausgezahlten Leistung aus dem gesetzlichen Rentenversicherungssystem betrug ab dem 1. Januar 2011 für eine alleinstehende Person $€$ 984,75 monatlich netto (von dem Nettobetrag ist der Beitrag für das AOWSystem schon abgezogen, Sociale Verzekeringsbank 2011).

Die Altersgrundrente bietet eine armutsvermeidende Einkommenssicherheit im Alter auch für vormals atypisch Beschäftigte, was zu der starken Verbreitung von Teilzeitarbeitsplätzen, dem sogenannten niederländischen »Teilzeitwunder", beigetragen haben dürfte (Klammer 2000: 317). Auch der Wechsel von einer abhängigen zu einer selbstständigen Beschäftigung bleibt hier ohne Auswirkung auf die Höhe der Alterssicherungsleistung aus dem staatlichen Kernsystem. Entscheidend ist hier lediglich die Dauer des Wohnsitzes in den Niederlanden. Charakteristisch für das niederländische System ist allerdings auch die hohe Bedeutung der zweiten Säule der Alterssicherung, der betrieblichen Altersversicherung. Rund 90\% der Beschäftigten in den Niederlanden sind durch derartige Alterssicherungsverträge erfasst. Hier sind unter Umständen Solo-Selbstständige aufgrund fehlender Betriebszugehörigkeit benachteiligt.

Wie das deutsche System ist das niederländische System der staatlichen Rentenversicherung umlagefinanziert, d. h. stark von der Anzahl der Beitragszahler abhängig, womit sich der demographische Wandel bzw. die Alterung der Bevölkerung als Problem darstellt. Unter dem Motto »Meedoen, meer werk, minder regels « (Mitmachen, Mehr Arbeit, weniger Regelungen) zielten die Reformen der letzten Jahren vor allem darauf, die Finanzierungsbasis des Alterssicherungssystem durch die Erhöhung der Arbeitspartizipation Älterer (d. h. der 55-65jährigen) zu erhöhen und steuerbegünstigte Frührentenmöglichkeiten zu begrenzen.

\section{Polen}

In Polen gibt es keine Altersgrundrente. Das erwerbs- und beitragsbezogene Altersrentenregelsystem bezieht auch Solo-Selbstständige außerhalb der Landwirtschaft und geringfügig Beschäftigte mit ein.

Das Rentensystem in Polen wurde im Jahr 1999 grundlegend reformiert. Die ersten Ansprüche aus dem neuen System konnten im Jahr 2009 geltend gemacht werden. Ziel der Reform war es, das System finanziell nachhaltig zu gestalten. Öffentliche Zuschüsse und der Beitrag der Arbeitgeber wurden reduziert. Es wurden Möglichkeiten frühzeitiger Verrentung abgebaut und eine stärkere Äquivalenz zwischen Beiträgen und Leistungen verankert, um einen deutlicheren Anreiz zu setzen in Arbeit zu bleiben. Für atypisch Beschäftigte mit geringen Einkommen liegen die Ersatzraten im neuen System niedriger. Als Resultat ist eine erhöhte Altersarmutsrate in der Zukunft zu befürchten (Golinowska et al. 2003).

Seit dem Inkrafttreten des Gesetzes zur Rentenversicherung 1999 besteht das System aus einem umlagefinanzierten und einem kapitalgedeckten Teil. Mit dem größten Teil der Beiträge wird das (mit einigen Ausnahmen) für alle Beschäftigten obligatorische Umlageverfahren finanziert. Die Höhe der Beiträge beläuft sich auf 19,5\% des Lohnes, die zu jeweils gleichen Teilen vom Arbeitgeber und vom Arbeitnehmer geleistet werden. 7,3\% des Arbeitnehmerbeitrages fließen dabei in offene Rentenfonds, die als Pflichtversicherung die zweite Säule des Systems darstellen. Sie werden

von privaten Anbietern verwaltet und finanzieren sich, neben den Beitragszahlungen, durch Wertpapierhandel und Zinszahlungen. Seit 2005 folgen die laufenden Rentenzahlungen zudem dem Prinzip der Preisindexierung, das heißt, dass sie von der Entwicklung der Verbraucherpreise abhängig sind (Schultewolter 2010).

Vor dem Hintergrund der anhalten- 


\begin{tabular}{|c|c|c|c|c|c|c|c|}
\hline & \multicolumn{2}{|c|}{ Grundsicherungssystem } & \multicolumn{4}{|l|}{ Regelsystem } & \multirow[t]{3}{*}{ Sonstiges } \\
\hline & \multirow[b]{2}{*}{$\mathrm{Ja}$} & \multirow[b]{2}{*}{ Nein } & \multicolumn{2}{|l|}{ Zugang zu Regelsystemen } & \multicolumn{2}{|l|}{ Bedingungen des Leistungsbezugs } & \\
\hline & & & Solo-Selbstständige & $\begin{array}{l}\text { Geringfügig } \\
\text { Beschäftigte }\end{array}$ & Mindestbeitragszeit & $\begin{array}{l}\text { Leistungsberechnung: Ab- } \\
\text { hängigkeit von vorherigem Ein- } \\
\text { kommen und Dauer der } \\
\text { Beschäftigung }\end{array}$ & \\
\hline DK & $\begin{array}{l}\text { X } \\
\text { (Volle Grundrente bei } \\
\text { 40 Jahren Wohnsitz) }\end{array}$ & & $\begin{array}{l}\text { Selektiv, freiwillige Weiterversi- } \\
\text { cherung für vormals min. } 3 \text { Jahre ab- } \\
\text { hängig beschäftigte Selbstständige }\end{array}$ & \begin{tabular}{|l} 
Teilweise (aus- \\
geschlossen sind \\
Beschäftigte mit Ar- \\
beitszeit $<9$ \\
Std/Woche)
\end{tabular} & Keine & $\begin{array}{l}\text { Unabhängig von vorherigen Ein- } \\
\text { kommen, hängt jedoch von den } \\
\text { Beiträgen (3 verschiedene Level, } \\
\text { je nach Art der Arbeit) \& der Ver- } \\
\text { sicherungsdauer ab. }\end{array}$ & $\begin{array}{l}\text { Betriebliche Alterssicherung } \\
\text { hat Deckungsgrad von } 90 \% \\
\text { bei abhängig Beschäftigten }\end{array}$ \\
\hline DE & & $\mathrm{x}$ & Selektiv & $\begin{array}{l}\text { Nein (bei Einkommen } \leq \\
400 € / \text { Monat) } \\
\end{array}$ & Kurz (5 Jahre) & $\mathrm{Ja}$ & \\
\hline IT & & $\mathrm{x}$ & $\mathrm{Ja}$ & Ja & $\begin{array}{l}\text { Versichert vor 1.1.1996: lang } \\
\text { (20 Jahre), danach: kurz } \\
\text { (5 Jahre) }\end{array}$ & \begin{tabular}{|l|} 
Versichert vor 1.1.1996: Ja, aber \\
Dauer der Erwerbstätigkeit ist \\
weniger entscheidend. Danach \\
Versicherte: Abhängig von Bei- \\
trägen während gesamter Erwerb- \\
szeit \\
\end{tabular} & \\
\hline NL & $\begin{array}{l}\text { X } \\
\text { (Volle Grundrente bei } \\
\text { 50 Jahren Wohnsitz) }\end{array}$ & & \multicolumn{4}{|c|}{ Kein Regelversicherungssystem neben der Grundrente siehe jedoch „Sonstiges“ } & $\begin{array}{l}\text { Betriebl. Alterssicherung hat } \\
\text { Deckungsgrad von }>90 \% \text { bei } \\
\text { abh. Beschäftigten }\end{array}$ \\
\hline \multirow[t]{2}{*}{ PL } & & $\mathrm{x}$ & Ja (außerhalb der Landwirtschaft) & \multirow[t]{2}{*}{ Ja } & \multirow{2}{*}{$\begin{array}{l}\text { Personen geboren vor } \\
\text { 1.1.1949: lang (M 20 Jahre, F } \\
\text { 15 Jahre), danach Geborene } \\
\text { haben keine Mindestbei- } \\
\text { tragszeit, entscheidend sind in- } \\
\text { dividuell gesammelte An- } \\
\text { wartschaften }\end{array}$} & \multirow{2}{*}{$\begin{array}{l}\text { Personen geboren vor 1.1.1949: Ja } \\
\text { danach Geborene: Abhängig von } \\
\text { Beiträgen }\end{array}$} & \\
\hline & & & $\begin{array}{l}\text { Auch für Selbstständige in der Land- } \\
\text { wirtschaft gibt es ein Versicherungssys- } \\
\text { tem. Der Transfer zwischen den ver- } \\
\text { schiedenen Versicherungssystemen (für } \\
\text { abhängig Beschäftigte, Landwirte, } \\
\text { Beamte) ist jedoch problematisch. }\end{array}$ & & & & \\
\hline UK & $\begin{array}{l}\text { X } \\
\text { (jedoch beitragsfinan- } \\
\text { ziert, freiwillige Bei- } \\
\text { träge können geleistet } \\
\text { werden) }\end{array}$ & & Nein & $\begin{array}{l}\text { Nein, (bei Arbeitneh- } \\
\text { mern Einkommen }< \\
119 € / \text { Woche) }\end{array}$ & Sehr kurz & Ja & \\
\hline
\end{tabular}

Tabelle 1: Alterssicherung und atypische Beschäftigung

Quellen: European Commission, Missoc (2010), Schulze Buschoff/Protsch (2008), OECD (2007), Golinowksa et al. (2003)

den Arbeitslosigkeit, der zu niedrigen Beschäftigungsquote und der zu kurzen Beschäftigungszeiten gelang es mit der Reform von 1999 nicht, die Finanzierung der Rentensysteme ausreichend $\mathrm{zu}$ stabilisieren. Eine nachhaltige Sicherung der Finanzierung wird auch dadurch erschwert, dass Bauarbeiter und Landwirte im Vergleich zu anderen Berufsgruppen durch Sonderregelungen im Rentenversicherungssystem nach wie vor großzügige Privilegien genießen (Schultewolter ebd.).

\section{Vereinigtes Königreich}

Im Vereinigten Königreich basiert das System der sozialen Sicherung auf den Grundprinzipien des Beveridge-Plans von 1942. Der Beveridge-Plan sieht vor, dass eine obligatorische beitragsbezogene Sozialversicherung eine Absicherung für den Einkommensausfall beim Eintritt der Risiken Alter, Krankheit, Mutterschaft, Arbeitslosigkeit und Verwitwung bieten sollte. Charakteristisch für den Beveridge-Plan ist die Orientierung an Erwerbsarbeit, verbunden mit sehr niedrigen Beiträgen und einer minimalen Grundsicherung für die gesamte Bevölkerung. Beveridge bezog in seinen Bericht die Selbstständigen bewusst mit ein - und setzte damit die Erkenntnis um, dass die Selbstständigen keineswegs eine homogene und wohlhabende Gruppe darstellen.

Selbstständige zahlen einen vergleichsweise niedrigen Beitragssatz zum nationalen Versicherungssystem (NICS = National Insurance Contributions). Im Gegensatz zu den abhängig Beschäftigten haben sie dafür auch keinen Anspruch auf Leistungen bei Arbeitslosigkeit und Arbeitsunfällen (Devetzi 1999: 47-48). Für geringfügig Beschäftigte besteht keine Versicherungspflicht in diesem System, sie können sich jedoch durch freiwillige Zahlungen weiterversichern.

Diejenigen, die Beiträge zum nationalen Versicherungssystem (NICS) zahlen, erwerben Ansprüche auf die staatliche Grundrente. Die Grundrente ist ein einkommensunabhängiger, einheitlich hoher Betrag, der jedes Jahr neu festgelegt wird. Die Leistungen des Grundrentensystems werden nur ausgezahlt, wenn Beiträge eine Mindestanzahl von Jahren geleistet wurden. Der volle Betrag, der keinesfalls armutsvermeidend ist, wird nur bei einer sehr langen Beitragszeit ausgezahlt, wobei allerdings Kinderbetreuung, Pflege und Arbeitslosigkeit an- gerechnet werden (Leschke 2009). Seit ihrer Einführung 1946 lag die Grundrente fast durchgehend auf einem Niveau unterhalb des Existenzminimums.

1977 wurde ein staatliches Rentenzusatzsystem eingeführt (»State Hearings Related Pensions Schemes: SERPS «, seit April 2002: "State Second Pension«), das zusätzlich zur einheitlichen Grundrente einkommensbezogene Zusatzrenten bereitstellen sollte (Hill 2003: 104). Von diesem staatlichen Zusatzrentensystem sind die Selbstständigen jedoch ausgenommen. Es besteht für sie auch keine Möglichkeit der freiwilligen Versicherung in diesem System. Für befristet Beschäftigte und Teilzeitbeschäftigte können Nachteile entstehen, weil die Leistung des Zusatzrentensystems deutlich von der Höhe des Einkommens und der

\section{Selbstständige haben nur mangelnden Zugang zu betrieblichen Altersvorsorgesystemen.}

Dauer der Beschäftigung abhängen. Von der Versicherungspflicht sind diejenigen befreit, die die Teilnahme an einem entsprechenden privaten Vorsorgesystem 
nachweisen können (»contracted-out«). Das Niveau der SERPS-Renten hängt zwar vom konkreten Einkommen ab, die höchstmögliche SERPS-Rente beträgt jedoch nur 20 Prozent des durchschnittlichen Arbeitseinkommens (Devetzi 2003: 412).

Dieses bescheidene Niveau der staatlichen Zusatzrenten, die steuerlichen Anreize zum »contracting-out « und das extrem niedrige Niveau der Grundrenten führen dazu, dass Betriebsrenten zur Aufrechterhaltung des bisherigen Lebensstandards eine immer wichtigere Rolle spielen. Selbstständige haben jedoch nur mangelnden Zugang zu betrieblichen Altersvorsorgesystemen. Da sie gleichzeitig auch vom staatlichen $\mathrm{Zu}-$ satzrentensystem und damit auch von der Möglichkeit des »contracting-out « ausgeschlossen sind, sind sie stärker als abhängig Beschäftigte auf die private Vorsorge zur Sicherung des Lebensstandards im Alter bzw. zur Vermeidung von Armut im Alter angewiesen.

\section{Fazit: Good Practices?}

Hinsichtlich der sozialen Sicherung von atypisch Beschäftigten besteht auf nationaler Ebene je nach Ausgestaltung der sozialen Sicherungssysteme unterschiedlicher Handlungsbedarf. Handlungsbedarf besteht besonders in Bezug auf die Absicherung Solo-Selbstständiger und geringfügig Beschäftigter. Diese Beschäftigten werden von den staatlichen Versicherungssystemen teilweise gar nicht erfasst oder deutlich schlechter behandelt als Beschäftigte in Normalarbeitsverhältnissen.

Insgesamt sind beitragsbezogene $\mathrm{Si}$ cherungssysteme mit Geringfügigkeitsgrenzen wenig geeignet, die mit der atypischen Beschäftigung verbundenen spezifischen Risiken (unstete und häufig niedrige Einkommen) abzufedern. Alternativ bzw. ergänzend dazu sollten Grundsicherungsmodelle weiterentwickelt werden. Staatliche Systeme, die auf dem Einwohnerprinzip basieren, Grundsicherungsmodelle integrieren und eine (zumindest teilweise) Steuerfinanzierung (bzw. eine Finanzierung mit Beiträgen, die nicht nur auf das Erwerbseinkommen, sondern auch auch andere Einkünfte erhoben werden) vorsehen, sind besser geeignet, eine Balance von atypischer Beschäftigung und Flexi- bilisierungsrisiken zu unterstützen (vgl. auch Hinrichs 2007 und Leschke 2006).

In Bezug auf die staatliche Alterssicherung ist entscheidend, ob trotz eines atypischen Erwerbsverlaufs ein zumindest armutsvermeidendes Renteneinkommen zu erwarten ist. Dies ist in den Niederlanden und in Dänemark der Fall,

\section{In Bezug auf die staatliche Alterssicherung ist entscheidend, ob trotz eines atypischen Erwerbsverlaufs ein armutsvermeidendes}

\section{Renteneinkommen zu} erwarten ist.

hier gewährleisten universelle Grundrentensysteme armutsvermeidende Einkommenssicherheit für alle Einwohner. In Dänemark wird das Grundrentensystem durch ein beitragsbezogenes Regelsystem ergänzt. In beiden Ländern bilden jedoch betriebliche Rentensysteme eine weitere wichtige Säule der Alterssicherung, von denen Solo-Selbstständige ohne Betriebsbindung ausgeschlossen sind.

In Italien und Polen gibt es keine Altersgrundrente, sondern beitragsbezogene Regelsysteme der Alterssicherung für alle Erwerbstätigen, einschließlich der Solo-Selbstständigen. In beiden Ländern besteht für atypisch Beschäftigte aufgrund ihrer häufig unsteten und niedrigen Einkommen die Gefahr von Altersarmut, in Polen insbesondere für nach dem 1.1.1949 geborene Personen, die im Gegensatz zu den früher geborenen keinen Anspruch mehr auf eine Mindestrente haben. In Polen kommt erschwerend hinzu, dass der Transfer von gesammelten Ansprüchen beim Wechsel zwischen Selbstständigkeit und abhängiger Beschäftigung problematisch ist.

Im Vereinigten Königreich sind mit Ausnahme der geringfügig Beschäftigten alle Erwerbstätigen im beitragsfinanzierten Grundrentensystem pflichtversichert. Daneben gibt es ein staatliches Zusatzrentensystem, von dem neben den geringfügig Beschäftigten auch die Solo-Selbstständigen ausgeschlossen sind. Die Höhe der Leistungen aus dem Grundrentensystem ist so niedrig, dass insbesondere für die von dem staatlichen Zusatzsystem ausgeschlossenen
Beschäftigten die Gefahr der Altersarmut besteht.

In Deutschland sind ein Großteil der Selbstständigen und geringfügig $\mathrm{Be}$ schäftigten nicht im beitragsbezogenen Regelalterssicherungssystem pflichtversichert. Die starke Orientierung am Äquivalenzprinzip ist für atypisch Beschäftigte mit häufig niedrigen und unsteten Einkommen nachteilig.

Im Ländervergleich ist auf der einen Seite in Dänemark und in den Niederlanden die soziale Absicherung atypisch Beschäftigter mit häufig universellem Zugang und hohen Nettoersatzquoten relativ umfassend gewährleistet. Dänemark und die Niederlande gelten als die Vorreiterländer, die, wenn auch unterschiedliche, »flexicurity«-Strategien umgesetzt haben.

Auf der anderen Seite steht Deutschland als Beispiel für ein Land, in dem die »flexicurity«-Strategie vergleichsweise wenig Anwendung findet. Das deutsche System der sozialen Sicherung ist aufgrund des selektiven Zuschnitts der Versicherungszweige und der starken Orientierung am Versicherungsgedanken (bzw. am Äquivalenzprinzip) nur unzureichend auf atypische Beschäftigungsverhältnisse und weitere Flexibilisierungstendenzen eingestellt. Während Standard-Beschäftigte hier recht hohe Schutzrechte genießen, sind für die atypisch Beschäftigten die Zugangsvoraussetzungen für die Versicherungssysteme hoch und die Leistungen relativ niedrig. Davon sind vor allem Frauen betroffen, da sie den Großteil der atypischen Beschäftigungen stellen.

Die politische Stoßrichtung der letzten Rentenreformen, in erster Linie den Beitragssatz zu stabilisieren und dafür Einschnitte bei den Leistungen in Kauf zu nehmen, führen in Deutschland auf längere Sicht zu einer deutlichen Erhöhung des Armutsrisikos für Rentner und Rentnerinnen. $\mathrm{Zu}$ einem erhöhten Altersarmutsrisiko tragen auch die Verschlechterung der rentenrechtlichen Berücksichtigung von Ausbildungszeiten oder von Zeiten des Bezugs von Arbeitslosengeld II bei. Treffen wird das erhöhte Altersarmutsrisiko vor allem die Beschäftigten im Niedriglohnbereich und die wachsende Zahl der atypisch und/ oder diskontinuierlich Beschäftigten. 


\section{Lehren für Deutschland?}

Die Umsetzung des auf der EU-Ebene propagierten Leibildes Flexicurity ist in Deutschland noch nicht überzeugend erfolgt. Mit den Hartz-Reformen aus den Jahren 2003 bis 2005 knüpfte die rot-grüne Bundesregierung an den Ansatz der Flexicurity an. Das FlexicurityKonzept bzw. eine stärkere Aktivierung von Arbeitssuchenden wurde umgesetzt, indem die rechtlichen Grundlagen einzelner Formen atypischer Beschäftigungsformen umfassend dereguliert wurden und so z. B. die Schaffung von geringfügiger Beschäftigung (Minijobs) und von Existenzgründungen aus der

\section{Eine OECD-Studie konstatiert, dass sich das Wohlstandsgefälle zwischen Arm und Reich in Deutschland in den letzten Jahren deutlich vergrößert hat.}

verhältnisse in Deutschland seit dem letzten Jahrzehnt vergleichsweise hoch. Die Industrie ist zwar nach wie vor vom männlich dominierten, sogenannten Normalarbeitsverhältnis geprägt. Im expandierenden Dienstleistungssektor haben aber im Vergleich zu anderen europäischen Ländern nur relativ wenige Beschäftigte eine unbefristete Vollzeitstelle (Eichhorst et al. 2010). Weiterhin ist der Anteil von Frauen in traditionellen Beschäftigungsverhältnissen aufgrund des lange dominierenden Male-Breadwinner-Modells unterdurchschnittlich. Die Entwicklung hin zu mehr Flexibilität auf dem Arbeitsmarkt trifft entsprechend eher Frauen als Männer. Der geringe Anteil von Frauen in Normalarbeitsverhältnissen ist in den letzten Jahren in Deutschland nochmals zurückgegangen (Eichhorst et al., ebd.). Deutlich gestiegen ist dagegen der Anteil von Frauen in atypischer Beschäftigung, d. h. insArbeitslosigkeit heraus (Ich-AGs) gefördert wurden. Weiterhin wurden stärkere Sanktionen beim Bezug von Arbeitslosengeld II (ALG II) z. B. bei Nichtaufnahme von Arbeitsangeboten in atypischer Beschäftigung eingeführt. Damit wird zwar die Flexibilität auf dem Arbeitsmarkt gefördert, dies geht aber nicht mit mehr Sicherheit für die betreffenden Beschäftigten einher, wie es eigentlich dem Flexicurity-Gedanken entsprechen würde. Elemente des `Forderns` sind sehr viel stärker ausgeprägt als Elemente des >Förderns`. Insgesamt haben die Reformen der letzten Jahre zu einer relativ ungleichen Verteilung von Risiken zugunsten der Beschäftigten in Normalarbeitsverhältnissen und zu ungunsten der atypisch Beschäftigten geführt. Die Segmentierung des Arbeitsmarktes geht einher mit der Zunahme sozialer Ungleichheit. Um dieser Entwicklung entgegen zu wirken, gilt es, die Durchlässigkeit zwischen den Segmenten zu fördern. Weiterhin ist eine Neujustierung der sozialen Sicherheit notwendig, damit nicht nur kontinuierliche, sondern auch flexible Verläufe und atypische Beschäftigungen adäquat abgesichert werden. Dies würde wesentlich zu einer größeren Chancengleichheit von Männern und Frauen beitragen.

Im europäischen Vergleich ist der Rückgang traditioneller Beschäftigungs- besondere der Frauen in Teilzeit, in geringfügiger Beschäftigung und in SoloSelbstständigkeit.

Die Ausweitung atypischer Beschäftigung ist im europäischen Vergleich in Deutschland besonders deutlich mit der Gefahr einer weiteren Prekarisierung verbunden. Eine 2008 veröffentlichte OECD-Studie konstatiert, dass sich das Wohlstandsgefälle zwischen Arm und Reich in Deutschland in den letzten Jahren deutlich vergrößert hat. Seit Mitte der 1990er Jahre haben sich die Löhne und Gehälter drastisch auseinander bewegt. »Von dieser Zunahme der Disparitäten waren die meisten (wenn auch nicht alle) Länder betroffen, wobei in Kanada und Deutschland in jüngster Zeit z. B. erhebliche Ausweitungen zu verzeichnen waren,..." (OECD 2008: 2). Weiterhin geht mit der Zunahme der Disparitäten eine insgesamt zähe Lohnentwicklung, d. h. eine im internationalen Vergleich unterdurchschnittliche Steigerung der Löhne, einher. Die Ausweitung atypischer Beschäftigung und insbesondere des Niedriglohnsektors bergen das Risiko der Zunahme sozialer Ungleichheit und der Prekarisierung.

Prekarierungs- und Altersarmutsrisiken werden durch die jüngsten Rentenreformen noch verstärkt. Das Ziel der
Reformen der Gesetzlichen Rentenversicherung (GRV) von 2001 und 2004 war es in erster Linie, die Beitragsbelastungen der künftigen Generationen soweit wie möglich zu begrenzen. Dafür wurden auch erhebliche Einschnitte bei den Leistungen in Kauf genommen. Weiterhin stellt sich bei der Neuregelung der Steuererleichterung bei privater Altersvorsorge über die sogenannte RürupRente bzw. die Riester-Rente angesichts der Zunahme von Geringverdienern die Frage, ob diese sich eine solche private Vorsorge überhaupt leisten können und ob nicht eher die ohnehin schon Privilegierten, nämlich die Besserverdienenden, davon profitieren (Niejahr 2006: 21).

Inzwischen hat sich die Ausrichtung der Diskussionen rund um die Weiterentwicklung der Rentenversicherung verschoben. In den aktuellen Diskussionen tritt die Frage des Leistungsniveaus wieder stärker in den Vordergrund (Thiede 2009: 355). Befürchtungen werden laut, dass das Leistungsniveau der GRV künftig zu einem deutlichen Anstieg der Altersarmut führen wird (Riedmüller und Willert 2008; Leiber 2009; Kerschbaumer 2010a). Insbesondere vor dem Hintergrund der Ausweitung atypischer und häufig niedrig entlohnter Beschäftigungsverhältnisse und zunehmend diskontinuierlicher Erwerbsbzw.- Versicherungskarrieren stellt sich die Frage, mit welchen Reformmaßnahmen der befürchteten Armut im Alter begegnet werden kann.

Fraglich ist dabei, ob ein Festhalten an der traditionellen deutschen Ren-

\section{Befürchtungen werden laut, dass das Leistungsniveau der GRV künftig zu einem deutlichen Anstieg der Altersarmut führen wird.}

tenpolitik, d. h. am reinen Sozialversicherungsprinzip mit einer starken Orientierung am Äquivalenzprinzip, den oben genannten Herausforderungen überhaupt gerecht werden kann. Als zukunftsfähiger und und überzeugender in Hinblick auf die Bewältigung der Probleme stellen sich Grundrentensysteme dar, z. B. die Systeme in Dänemark oder in den Niederlanden.

In Dänemark bietet eine steuerfi- 
nanzierte Volks- bzw. Altersgrundrente armutsvermeidende Einkommenssicherheit für alle Einwohner, die ihren Wohnsitz ausreichend lange im Land hatten. Die Mitgliedschaft hängt allein vom Wohnen in Dänemark und nicht davon $\mathrm{ab}$, ob der/die Versicherte eine Beschäftigung ausgeübt hat. Auch der niederländische Staat stellt allen Einwohnern ab dem 65. Lebensjahr eine staatliche Grundsicherung zur Verfügung, die nur auf das Wohnen im Land abgestellt ist und bei der die Erwerbstätigkeit keine Rolle spielt. In beiden Ländern haben auch Einwohner, die atypisch, diskontinuierlich oder auch gar nicht beschäftigt waren, Anspruch auf eine staatliche Altersgrundrente. In den Niederlanden gibt es bei der staatlichen Altersgrundsicherung keine Bedürftigkeitsprüfung, in Dänemark kann die Höhe der Leistungen der staatlichen Grundrente gekürzt werden, wenn der Empfänger weitere Einkommen bezieht, die über bestimmten Grenzen liegen. In beiden Ländern ist das Niveau der Leistungen armutsvermeidend. Vor allem das niederländische Modell könnte als Vorbild dienen, da es wie das deutsche System umlagefinanziert ist. Umlagefinanzierungen haben sich als recht krisenfest erwiesen. In den Niederlanden werden die Beiträge zur Altersgrundsicherung auf viele Einkunftsarten und nicht nur auf die Erwerbseinkommen erhoben, diese breitere Finanzierungsbasis stärkt die Nachhaltigkeit des Systems. Nichtsdestotrotz ist die Finanzierungsbasis des niederländischen System, wie andere umlagefinanzierte Systeme auch, von dem demographischen Wandel und der Alterung der Bevölkerung bedroht. Um die Zahl der Beitragszahler zu erhöhen, ist der niederländische Staat bemüht, das durchschnittliche, tatsächliche Renteneintrittsalter zu erhöhen und Frühverrentungsmöglichkeiten zu begrenzen.

Die Einführung einer Grundrente nach dänischem oder niederländischen Vorbild in Deutschland würde einen deutlichen System- und damit Paradigmenwechsel bedeuten. Dies würde der in der Wohlfahrtsstaatsforschung seit den 1990er Jahren dominierenden Vorstellung von der »Pfadabhängigkeit « sozialstaatlicher Reformen widersprechen. Nach dieser Vorstellung kann sozialpolitischer Wandel nur »inkrementell«, d. h. in kleinen Schritten und marginal erfolgen, da von historisch eingeschla- genen Pfaden nicht abgewichen wird. Argumentiert wird, dass insbesondere der deutsche, konservative Wohlfahrtsstaat reformträge sei. Doch gerade in Deutschland können jüngere Reformen wie die Einführung der Riester-Rente und die Hartz-Reformen als Beleg dafür gelten, dass unter bestimmten Rahmenbedingungen Pfadabweichungen und Paradigmenwechsel in der Reformpolitik möglich sind (Jochem 2011). Gegen einen Systemwechsel in der Deutschen Rentenversicherung spricht allerdings ein gewichtiges Argument: der Bestandsschutz, das Problem bereits erworbener Ansprüche und die Frage der Überleitung zu einem neuen System stellen große Hürden dar.

Sicherlich finden sich auch für die Beibehaltung der tradierten deutschen Rentenpolitik gute Argumente, so etwa die paritätische Beitragszahlung und die Einbeziehung der Versicherungsnehmer in die Organisation und Verwaltung der Systeme. So wird einerseits vertreten, dass die deutsche Sozialversicherung zukunftsfähig sei (Bogedan/ Leiber 2009: 400). Andererseits dürfte unumstritten sein, dass das System in seiner derzeitigen Ausgestaltung angesichts des aufgezeigten Problemdrucks reformbedürftig ist.

Offensichtlich ist, dass das Organisationsprinzip der Rentenversicherung in Deutschland der Lebensrealität eines Großteils der Bevölkerung zuwiderläuft. Während es mit der Orientierung am Versicherungsgedanken und der Anwendung des Äquivalenzprinzips auf dauerhafte und kontinuierliche Vollzeitbeschäftigung setzt, sind hohe Flexibilität, Arbeitsmarktmobilität und Brüche charakteristisch für heutige Erwerbsbiographien. Zur adäquaten Einbeziehung atypischer Beschäftigungsformen in die sozialen Sicherungssysteme ist eine Erwerbsverlaufsperspektive nötig, mitsamt der Einbeziehung von Übergängen im Lebensverlauf, bzw. von unsteten Einkommen und Brüchen in der Erwerbsbiographie. In der Sicherung dieser Risiken gibt es noch erhebliche Defizite.

Überlegungen zur Absicherung unsteter Einkommensverläufe wurden mit der Idee sogenannter »flexibler Rentenanwartschaften « bereits vor ein paar Jahren in Form von überzeugenden Mo- dellrechnungen vorgelegt (Langelüddeke et a. 1999; Thiede 2000). Mit flexiblen Anwartschaften würde man während Phasen der Vollzeitbeschäftigung Anwartschaften erwerben, welche bei Phasen verminderter Anwartschaften

\section{ur adäquaten} inbeziehung atypischer Beschäftigungsformen in die ozialen Sicherungssysteme ist eine Erwerbsverlaufserspektive nötig.

ausgeglichen werden würden. Auf diese Weise würden die negativen Wirkungen diskontinuierlicher Erwerbsverläufe abgemildert. Unabhängig von der Höhe des Beitrags, den man erbracht hat, erhält man einen zusätzlichen Entgeltpunkt. Die zusätzlichen Anwartschaften sind auf fünf Entgeltpunkte begrenzt und können von den Versicherten zur Schließung von Sicherungslücken flexibel eingesetzt werden.

Die Zunahme atypischer Beschäftigungsformen und unsteter Erwerbsbiographien stellt sich auch in Bezug auf die Arbeitslosenversicherung als $\mathrm{He}$ rausforderung zur Entwicklung neuer sozialer Sicherungselemente dar. Um die soziale Sicherung zu erweitern, ist der Vorschlag zur Einrichtung persönlicher Entwicklungskonten als Ergänzung der tradierten aktiven Arbeitsmarktpolitik entwickelt worden (Schmid 2008). Dahinter steht die Idee der Erweiterung der Arbeitslosen- zur Beschäftigungsversicherung. Derzeit sichert die Arbeitslosenversicherung nur das Einkommensrisiko bei Arbeitslosigkeit ab, mit der Beschäftigungsversicherung würden auch die oftmals riskanten Übergänge im Erwerbsverlauf berücksichtigt. Weiterhin könnte aktuellen Anforderungen, wie dem Risiko der Erosion der Bildung im Erwerbsverlauf, entsprochen werden. Die Beschäftigungsversicherung zielt somit auf die Eindämmung von Exklusionsgefahren am Arbeitsmarkt, von denen vor allem atypisch Beschäftigte betroffen sind.

Eine der größten und zugleich schwierigsten Herausforderungen für die deutsche Rentenversicherung besteht in der konkreten Ausgestaltung eines längst überfälligen Reformschrittes, nämlich 
der Ausweitung der Pflichtversicherung auf alle Erwerbstätigen - und damit auch auf die geringfügig Beschäftigten und auf alle Gruppen von Selbstständigen. Vergleicht man die verschiedenen Formen atypischer Beschäftigung, dann bestehen die größten Sicherungslücken derzeit für die nicht versicherungspflichtigen Solo-Selbstständigen und für die ebenfalls nicht versicherungspflichtigen geringfügig Beschäftigten.

\section{Geringfügige Beschäftigung}

Angesichts einer dramatischen Fehlentwicklung durch die Ausweitung von "Minijobs « ist dem deutschen Frauenrat zuzustimmen, der die Abschaffung der Geringfügigkeitsgrenze und die Einführung einer umfassenden Sozialversicherungspflicht fordert (Deutscher Frauenrat 2010). Derzeit bedeutet die große

\section{Minijobs führen zu Armutsrenten: Betroffen sind davon in erster Linie Frauen.}

Zahl der Minijobs (ca. sieben Millionen) deutliche Einnahmeverluste für die Sozialversicherungsträger. Im deutschen, deutlich am Äquivalenzprinzip orientierten Rentensystem ist die Gefahr der Altersarmut für geringfügig Beschäftigte gravierend: Minijobs führen zu Armutsrenten. Betroffen sind davon in erster Linie Frauen, deren Durchschnittsrente ohnehin geringer als die der Männer ist. Die öffentlichen Sozialausgaben steigen entsprechend an, z. B. durch Inanspruchnahme der Hinterbliebenenrente oder einer Grundsicherung. Minijobs sind vor allem für immer mehr Frauen die alleinige Verdienstquelle. Dadurch sind diese Arbeitnehmer/innen aber nicht gegen Arbeitslosigkeit versichert und auch in der Gesetzlichen Kranken- und Rentenversicherung nicht eigenständig abgesichert. Vielen bleibt nur die Mitversicherung bei dem Ehepartner/der Ehepartnerin. Folge davon ist eine dauerhafte existenzielle Abhängigkeit von dem/der Partner/in bzw. von staatlichen Transferleistungen, in der Erwerbsphase und im Alter. Durch »billige « Minijobber/innen, geraten auch Tariflöhne weiter unter Druck, insbesondere nachdem 2003 die ursprüngliche Begrenzung auf eine zulässige Höchstarbeitszeit weggefallen ist. Weiterhin ist davon auszugehen, dass reguläre Beschäftigungsverhältnisse durch Minijobs in erheblichem Maße verdrängt werden.

\section{Solo-Selbstständigkeit}

Durch das solidarische, umlagefinanzierte staatliche System der Sozialen Sicherung werden die Selbstständigen nur teilweise, meist im Rahmen von Sonderregelungen, erfasst. Für den Großteil der Selbstständigen besteht keinerlei Sozialversicherungspflicht zur Altersvorsorge, obwohl sie vielfach ähnlich wie Arbeitnehmer auf Erwerbseinkommen angewiesen sind und häufig geringere und unregelmäßige Einkommen erzielen als diese. Eine Versicherungspflicht besteht wiederum dann, wenn ein Arbeitsverhältnis die Kriterien einer vermuteten

Scheinselbstständigkeit erfüllt. Mit der Begrenzung der Pflichtversicherung auf wenige Sondergruppen Selbstständiger (bzw. Scheinselbstständiger) stellt Deutschland im europäischen Vergleich eine Besonderheit dar. Denn in der Mehrzahl der europäischen Länder werden die Selbstständigen durch die staatlichen Pflichtversicherungssysteme systematisch erfasst.

Die Schaffung neuer Sonderregelungen für bestimmte Gruppen, wie z. B. die Einführung der Künstlersozialversicherung oder der spezifischen sozialversicherungsrechtlichen Regelungen des Existenzgründungszuschusses (>Ich$A G<)$, setzen die unsystematische und uneinheitliche Einbeziehung von Minderheitsgruppen fort. Dadurch werden auf der einen Seite zwar Privilegien für die Sondergruppen, auf der anderen Seite jedoch auch neue Hürden, Ausgrenzungen und Benachteiligungen für die nicht berücksichtigten Gruppen geschaffen.

Die unsystematische und partikuläre Erfassung Selbstständiger durch das Sozialversicherungsrecht führt zu einer Verstärkung der sozialen Risiken für einen wachsenden Teil der Selbstständigen. Statt weiterer Sonderregelungen - etwa für bestimmte Berufsgruppen, für von der Arbeitsagentur geförderte Selbstständige oder für Scheinselbstständige - sollte der generelle soziale Schutzbedarf Selbstständiger anerkannt werden. Und analog zu den Regelungen bei abhängig Beschäftigten sollten möglichst universelle Regelungen geschaffen werden (Betzelt 2004: 34). Gefordert wird in Deutschland deshalb vielfach die obligatorische Einbeziehung aller Selbstständigen in die gesetzlichen Sozialversicherungssysteme, insbesondere in das System der gesetzlichen Rentenversicherung (Bieback 2001; Betzelt/Fachinger 2004; Schulze Buschoff 2005).

Mit der geforderten Einbeziehung der Selbstständigen in die gesetzlichen Sozialversicherungssysteme stellt sich jedoch das Problem der Beitragsgestaltung. Durch die Einbeziehung aller Selbstständigen in die gesetzliche Sozialversicherung würde eine stärkere Entkoppelung des sozialen Schutzes vom Arbeitsverhältnis bzw. vom Arbeitnehmerstatus bewirkt, wodurch das Prinzip der paritätischen Beitragszahlung in Frage gestellt wird. Die Ausweitung der Sozialversicherungen über den Kreis der abhängig Beschäftigten hinaus führt dazu, dass vielen Versicherten ein Arbeitgeber ‘fehlt , der einen Teil der Versicherungsbeiträge übernehmen könnte.

In dem derzeit geltenden beitragsbezogenen Rentenversicherungssystem Deutschlands sind nur rund ein Viertel aller Selbstständigen in der gesetzlichen Rentenversicherung pflichtversichert. Bestehen keine besonderen Konditionen wie z. B. bei der Künstlersozialkasse (KSK), dann müssen die in der gesetzlichen Rentenversicherung freiwillig oder pflichtversicherten Selbstständigen ihren Beitrag vollständig selbst aufbringen. Wegen dieser Rahmenbedingungen sind die Beiträge relativ hoch. Die derzeit geltenden Regelungen in den gesetz-

\section{Statt weiterer Sonder- regelungen sollte der generelle soziale Schutzbedarf Selbst- ständiger anerkannt werden.}

lichen Sozialversicherungssystemen führen insbesondere für pflichtversicherte Selbstständige im unteren Einkommensbereich $\mathrm{zu}$ einer erheblichen relativen Belastung.

Die Dienstleistungsgewerkschaft ver. di, die hauptsächlich im Medienbereich tätige Selbstständige als Mitglieder rekrutiert und organisiert, propagiert 
analog zur Künstlersozialkasse, die Beteiligung der Auftraggeber an den Sozialversicherungsbeiträgen. Ein Auftraggeber-Anteil könnte in Form eines Honoraranteils einbezogen werden, der wie bei der Mehrwertsteuer als Sozialversicherungsbeitrag in die Rechnung eingetragen und auf den Endpreis der Arbeitsleistung aufgeschlagen würde. Der/die Selbstständige wäre dann für die Abführung der Sozialversicherungsbeiträge selbst verantwortlich. Eine weitere Variante wäre ein Zuschuss aus Steuermitteln zu den Sozialversicherungsbeiträgen bei kleinen Einkommen, die sich der Staat durch eine AuftraggeberAbgabe rückerstatten lassen könnte (ver. di 2005: 134). Denkbar wäre auch die finanzielle Entlastung der Selbstständigen mit niedrigen Einkommen bei der Beitragsgestaltung durch Zuschüsse aus allgemeinen Steuermitteln, z. B. in Form eines Freibetrages oder eines Beitragserlasses (Betzelt 2004).

Bei der Beitragsgestaltung Selbstständiger in der tradierten gesetzlichen Rentenversicherung gilt es, die schwierige Balance zwischen zwei Zielen zu meistern: Zum einen sollten die Beitragslast insbesondere für >kleine Selbstständige erschwinglich und die Konditionen der Versicherung lohnenswert sein. Zum anderen sollte die Finanzierungsbasis der gesetzlichen Sozialversicherung nicht geschwächt werden und eine Neuregelung sollte nicht zu Lasten der anderen Beitragszahler/innen gehen.

Im Einzelnen sind folgende Handlungsoptionen zur Vermeidung von Altersarmut atypisch Beschäftigter zu nennen, die 1. auf das Erwerbssystem, bzw. die Erwerbsbeteiligung und die Arbeits- und Einkommensbedingungen, 2. auf die Reformierung des bestehenden Rentenversicherungssystems und 3. auf einen Systemwechsel abzielen.

1. Handlungsoptionen, die auf das Erwerbssystem, bzw. die Erwerbsbeteiligung und -bedingungen abzielen:

- Auf der Seite der Arbeitsmarktpolitik: Weg von der der Niedriglohnstrategie

- Angleichung der Verdienste in Ostund Westdeutschland

- Maßnahmen zur Erhöhung der Erwerbsbeteiligung, vor allem von Frauen und Älteren

- Equal pay, Gleicher Lohn für gleichwertige Arbeit, Beseitigung der Lohndifferenzen zwischen Frauen und
Männern

- Maßnahmen zur Vereinbarkeit von Familie und Beruf

- Reform der Arbeitslosenversicherung zur Beschäftigungsversicherung

- Beschäftigungsfähigkeit bestimmter Gruppen verbessern/ in Fort- und Weiterbildung investieren, Förderung der Qualifizierung und beruflichen Weiterbildung Benachteiligter

- Einführung eines flächendeckenden gesetzlichen Mindestlohnes, Stärkung der Tarifbindung und angemessene Löhne

- Abschaffung der Geringfügigkeitsgrenze (und damit der Minijobs) verbunden mit der Einführung einer Sozialversicherungspflicht ab der ersten Arbeitsstunde, bzw. ab dem ersten Euro Verdienst. (Bei Beibehaltung einer Geringfügigkeitsgrenze ist die (Wieder-) Einführung einer Grenze für die zulässige Arbeitszeit in Stunden unerlässlich.)

- Abschaffung des Ehegattensplittings im Steuerrecht

2. Handlungsoptionen bezüglich des Rentenversicherungssystems, Reform des bestehenden Systems:

- Abkehr vom starren Versicherungsgedanken bzw. vom Äquivalenzprinzip, so dass mehr Spielräume zur Umverteilung zugunsten von Geringverdienern geschaffen werden.

- Umverteilung zugunsten von Geringverdienern z. B. in Form der Wiederbelebung der in der GRV 1992 abgeschafften "Rente nach Mindesteinkommen ", die unterdurchschnittliche Einkommen bei langjährig Versicherten bei der Ermittlung der Entgeltpunkte erhöhte

- Anerkennung von Zeiten der Berufsausbildung als Rentenversicherungsjahre

- Anhebung des Renteneintrittsalters auf 67 Jahre erst dann, wenn eine deutliche Erhöhung des tatsächlichen durchschnittlichen Renteneintrittsalters verzeichnet werden kann

- Bessere rentenrechtliche Bewertung von Zeiten einer längeren Arbeitslosigkeit

- Flexible Anwartschaften

- Einbeziehung der bislang nicht pflichtversicherten Selbstständigen in die gesetzliche Rentenversicherung

- Versicherungspflicht für alle Erwerbstätigen
3. Handlungsoption bezüglich des Versicherungssystems, Systemwechsel:

- Einführung einer armutsvermeidenden Grundrente

Abschließend bleibt zu betonen, dass bei der Gestaltung der Renten- und der Arbeitsmarktpolitik eine stärkere Koordinierung erfolgen sollte entlang einer übergeordneten und gemeinsamen Zielrichtung, nämlich der Sicherung existenzsichernder und angemessener Löhne im Erwerbsleben und armutsfester Renten im Alter. Die Flexibilisierung der Arbeitsmärkte und weitere Reformen des Rentensystems dürfen nicht weiterhin zulasten der sozialen Sicherheit der Beschäftigten erfolgen. In der Kombination von sozialer Sicherheit und Flexibilität liegt die zentrale Dimension armutsfester und zukunftsfähiger Systeme.

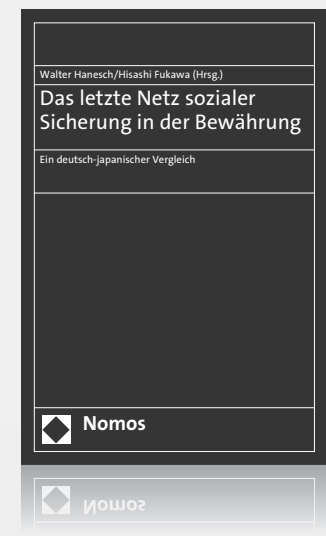

\section{Das letzte Netz sozialer Sicherung in der Bewährung}

Ein deutsch-japanischer Vergleich

Herausgegeben von Walter Hanesch und Hisashi Fukawa

2012, 319 S., brosch., 59,- $€$ ISBN 978-3-8329-5965-4

Www.nomos-shop.de/12916

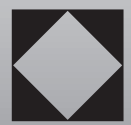

Nomos 
Aerts, Monique (2005): "The Dutch social insurance system for self-employed." In: Wissenschaftszentrum Berlin für Sozialforschung (Hg.): WZB discussion paper, SP I 2005-111.

\section{Arrowsmith, James, Keith Sisson and Paul} Margison (2004): What can "benchmarking" offer the open method of co-ordination? In: Journal of European Public Policy 11 (2): 311-328. Special issue "The open method of co-ordination in the European Union«.

Betzelt, Sigrid (2004): Konzeptvorschlag zur sozialen Altersabsicherung Selbstständiger. Gutachten im Auftrag des Projekts mediafon der Vereinten Dienstleistungsgewerkschaft (ver.di). Bremen

\section{Betzelt, Sigrid, und Uwe Fachinger (2004):} Jenseits des »Normalunternehmers«: Selbst ständige Erwerbsformen und ihre soziale Absi cherung, Zeitschrift für Sozialreform 3: 21-30.

Bieback, Karl-Jürgen (2001): Soziale Absicherung neuer Selbständiger. In: WSI-Mitteilungen 53, Heft 12, S. 810-817.

Bieber, Ulrich (2003): "Niederlande«, in: VDR, Verband deutscher Rentenversicherungsträger (Hg.): Rentenversicherungen im internationalen Vergleich. DRV-Schriften, Bd. 45, Bad Homburg: WDV Wirtschaftsdienst: 137-166.

Bogedan, Claudia/ Leiber, Simone (2009): Fit für die Zukunft: Vorschläge für eine moderne Sozialversicherung. In: WSI-Mitteilungen 7/2009, S. 400-401.

Bregaard, Thomas/Larsen, Fleming/Madsen, Per K./Rasmussen, Stine (2009): "Flexicurity und atypische Beschäftigung in Dänemark." In: WSI-Mitteilungen 1/2009, 31-38.

Brzinsky-Fay Christian; Protsch, Paula und Karin Schulze Buschoff (2007): Atypische Beschäftigung - Umfang, Dynamik und soziale Sicherung im europäischen Vergleich. Unveröffentlichtes Konferenzpapier zur 5. Internationalen ISSA-Forschungskonferenz über soziale Sicherheit am 5. bis 7. März in Warschau.

Deutscher Frauenrat (2010): "Minijobs - Wege in die Armut. Kann sich unsere Gesellschaft Minijobs leisten? "Positionspapier des Deutschen Frauenrates zu Minijobs beschlossen auf der Mitgliederversammlung 2010.

\section{Deutscher Gewerkschaftsbund (DGB) (2011)}

Niedriglohn und Lohndumping im Verleihgewerbe. DGB Abteilung Arbeitsmarktpolitik aktuell Nr. 2/ Februar 2011

Devetzi, Stamatia (2003): Großbritannien. In: VDR, Verband deutscher Rentenversicherungs träger (Hg.): Rentenversicherungen im internationalen Vergleich. DRV-Schriften, Bd. 45.Bad Homburg: WDV Wirtschaftsdienst, S. 391-417.

Dienel, Christiane und Sabine Overkämping (2010): Der Vertrag von Lissabon und die europäische Sozialpolitik. In: Leiße, Olaf (Hrsg.) Die Europäische Union nach dem Vertrag von Lissabon. VS Verlag, Wiesbaden. S. 176-195

Eichhorst, Werner/Kuhn, Andrea/Thode, Eric/Zenker, Rosemarie (2010): Traditionelle Beschäftigungsverhältnisse im Wandel. Benchmarking Deutschland: Normalarbeitsverhältnis auf dem Rückzug. Bertelsmann Stiftung, Gütersloh.
Eigmüller, Monika (2010): Vom Nationalstaat lernen? Möglichkeiten und Grenzen der Analogiebildungen zwischen nationaler und europäischer Sozialpolitikentwicklung. In:

Eigmüller, Monika und Steffen Mau (Hrsg.): Gesellschaftstheorie und Europapolitik. Sozialwissenschaftliche Ansätze zur Europaforschung. VS Verlag, Wiesbaden. S. 353-379

European Commission (2004): Employment in Europe, Brussels.

European Commission (2006): Modernising labour law to meet the challenges of the 21st century, European Commission Green Paper, Brussels.

European Commission (Missoc) (2010): Comparative Tables on Social Protection in the 25 Member States of the European Union, in the European Economic Area and in Switzerland Situation on 1 January 2010. Brüssel

Europäische Kommission (2010): Europa 2020. Eine Strategie für intelligentes, nachhaltiges und integratives Wachstum. Mitteilung der Kommission. Brüssel, den 3.3. 2010, KOM (2010) $2 \mathrm{O} 2 \mathrm{O}$

Frankfurter Allgemeine Zeitung (FAZ) (2011): Junge Renter in Italien. Onlineartikel: http:// www.faz.net/s/RubC9401175958F4DE28E 143E68888825F6/Doc E2BE8483064A74CF88 Eo94FFFE3C7EB6C ATpl Ecommon Scontent. html, abgerufen am 12.02.2011.

Giesecke, Johannes und Martin Groß (2005): Befristete Beschäftigung. In: WSI-Mitteilungen 5/2006. S. 247-261.

Girndt, Cornelia (2008): Die EugH-Urteile. In: Mitbestimmung 7/8. S. 22

Golinowksa, Stanislawa; Pietka, Katarzyna; Sowada, Christoph und Maciej Zukowsky (2003): Study on the Social Protection Systems in the 13 Applicant Countries - Poland, Country Study. Gesellschaft für Versicherungswissenschaft und -gestaltung e. V.

Hill, Michael (2003): Understanding Social Policy. 7. Aufl. Oxford: Blackwell Publishing.

Hinrichs, Karl (2007): Do we need better basic protection in old age to make flexible labour markets more palatable? A modest plea for universal tax-financed pensions. Paper presented on the 5 th International Research Conference on Social Security, Warsaw, 5-7 March 2007

Jochem, Sven (2011): Skandinavische Arbeitsund Sozialpolitik. Vorbilder für einen vorsorgenden Staat. Studie für die Friedrich-EbertStifung, Internationale Politikanalyse, Berlin.

Kerschbaumer, Judith (2010a): Die Frau lebt nicht vom Mann allein. Auswirkungen von Lohnungleichheit auf die Alterssicherung von Frauen. In: Humboldt chancengleich, Juni 2010 S. 6-8.

Kerschbaumer, Judith (2010b): ver.di-Stellung nahme zum EU-Grünbuch Rente. Sopo-aktuell vom 29.November 2010.

Klammer, Ute (2000): Auf dem Weg zu mehr Flexicurity in Europa. WSI-Mitteilungen 5/2000, S. 313-321.
Langelüdekke, Anne; Rabe, Birgitta; Thiede, Reinhold (1999): Flexibel Anwartschaften und Anwartschaftszeiten. In: Die Angestelltenversicherung 1, S. 7-13.

Leiber, Simone (2009): Armutsvermeidung im Alter: Handlungsbedarf und Handlungs optionen. WSI-Diskussionspapier Nr. 166, Düsseldorf.

Leschke, Janine (2006): Are unemployment insurance systems in Europe adapting to new risks arising from non-standard employment? Vortrag zur 27. Konferenz der International Working Party on Labour Market Segmentation (IWPLMS), 14.-16. September 2006

Leschke, Janine; Schmid, Günther und Dorit Griga (2006): On the Marriage of Flexibility and Security:Lessons from the Hartz-reforms in Germany. WZB-Discussion paper SP I 2006 108.

Leschke, Janine (2009): Flexible Erwerbsverläufe und Sozialversicherung. In WSI-Mitteilungen 7/2009, S. 383-390.

Madsen, Per Kongsh $\varnothing \mathbf{j}$ (2002): The Danish Model of »Flexicurity «-A Paradise with some Snakes. Conference Paper, European Foundation for the Improvement of Living and Working Conditions Interactions between Labour Market and Social Protection. Brussels May 16, 2002

Niederlandeweb (2011): Rente in den Niederlanden, Onlineartikel, http://www.unimuens ter.de/HausDerNiederlande/zentrum//20/20. html, (abgerufen am 21.02.2011).

Niejahr, Elisabeth (2006): Kollegen zweiter Klasse. In: Die ZEIT, Nr. 10, 02.03.2006, S. $21 \mathrm{f}$.

OECD (2007): Pensions at Glance, Public Policies across OECD countries, 2007 Edition. Pdf-Datei abrufbar unter www.oecd.org/publications. Abgerufen am 01.12.2010.

OECD (2008): Mehr Ungleichheit trotz Wachstum? Einkommensverteilung und Armut in OECD-Ländern. Zusammenfassung. http:// www.oecd.org/dataoecd/45/26/41525363.pdf (05.02.10)

Preunkert, Jenny (2009): Chancen für ein soziales Europa? Die offene Methode der Koordinierung als neue Regulierungsform. VS Verlag für Sozialwissenschaften, Wiesbaden.

Rat der Europäischen Union (2005): Entscheidung des Rates vom 12. Juli 2005 für beschäftigungspoltische Maßnahmen der Mitgliedstaaten. http://eurlex.europa.eu/LexUriServ/ LexUriServ.do?uri=OJ:L:2005:255:0009:0010:D E:PDF (15.02.10).

Rat der Europäischen Union (2008): Umsetzung der gemeinsamen Grundsätze für den Flexicurity-Ansatz im Rahmen des Zyklus 2008-2010 der Lissabon-Strategie - Bericht der Flexicurity-Mission (17047/08). http:// ec.europa.eu/social/BlobServlet?docld $=1515 \&$ la ngld=de (05.01.11)

Riedmüller, Barbara/Willert, Michaela (2008): Die Zukunft der Alterssicherung. Analyse und Dokumentation der Datengrundlage aktueller Rentenpolitik, Gutachten im Auftrag der HansBöckler-Stiftung, Düsseldorf. 


\section{Literatur}

Schmid, Günther (2000): Von standardisierten zu variablen Arbeitsverhältnissen. In: Kocka, Jürgen/ Offe, Claus (Hrsg.): Geschichte und Zukunft der Arbeit. Campus Verlag, Frankfurt a.M., New York, S. 269-293.

Schmid, Günther (2008): Entgrenzung der Erwerbsarbeit - Erweiterung der sozialen Sicherheit. In: WSI-Mitteilungen 7/2008, S. 358-364.

Schmid, Günther (2010): Non-Standard Employment and Labour Force Participation: A Comparative View of the Recent Development in Europe. IZA discussion paper No. 5087.

Schmid, Günther /Protsch, Paula (2009): Wandel der Erwerbsformen in Deutschland und Europa. WZB discussion paper, SP I 2009-505.

Sociale Verzekeringsbank (2011): AOW pensioen, AOW-bedragen, Onlineartikel, http://www. svb.nl/int/nl/aow/hoogte_aow/bedragen/ index.jsp, (abgerufen am 21.02.2011).

Schultewolter, Daniel (2010): Sozialstaat und Rentensystem in Polen. Eine Bestandsaufnahme. Länderbericht der Konrad-AdenauerStiftung e. V., Sankt Augustin, 8. Juni 2010.
Schulze Buschoff, Karin (2004): Neue Selbstständigkeit und wachsender Grenzbereich zwischen selbstständiger und abhängiger Erwerbsarbeit - europäische Trends vor dem Hintergrund sozialpolitischer und arbeitsrechtlicher Entwicklungen. WZB-discussionpaper SP I 2004-108, Berlin.

Schulze Buschoff, Karin (2005):Von der Scheinselbstständigkeit zur Ich-AG - neue sozialpolitische Weichenstellungen? In: Zeitschrift für Sozialreform (ZSR), Nr. 1/2005: 64-93.

Schulze Buschoff, Karin/Schmidt, Claudia (2005): »Die Status-Mobilität der SoloSelbstständigen und ihre soziale Sicherung im europäischen Vergleich.« In: Zeitschrift für Arbeitsmarktforschung (ZAF) 4, 531-557.

Schulze Buschoff, Karin/Protsch, Paula (2008): „Die soziale Sicherheit von (a-)typisch Beschäftigten im europäischen Vergleich." In: Internationale Revue für Soziale Sicherheit (ISSR). 4/2008, S. 51-73.

Tangian, Adranik (2005): Monitoring Flexicurity Policies in the EU with Dedicated Compositelndicators. WSI-Discussionspapier Nr. 137. Düsseldorf: Hans-Böckler-Stiftung.
Thiede, Reinhold (2000): Flexible Anwartschaften: Modell zur Modernisierung des Rentensystems, in: Soziale Sicherheit 2, S. 48-52.

Thiede, Reinhold (2009): Mindestsicherungselemente in der gesetzlichen Krankenversicherung? Flexible Erwerbsverläufe und Sozialversicherung. In WSI-Mitteilungen 7/2009, S. $355-362$

Ver.di, Vereinte Dienstleistungsgewerkschaft, Abteilung Sozialpolitik/Gesundheitspolitik (2005): Sozialpolitische Informationen. Daten/ Fakten/ Hintergründe, 1. Halbjahr 2005.

Wilthagen, Ton (1998): Flexicurity: A New Paradigm for Labour Market Policy Reform. In: Wissenschaftszentrum Berlin für Sozialforschung (Hg.): WZB discussion paper, FS-I 98-202.

Wilthagen, Ton/Tros, Frank (2004): The Concept of ,Flexicuritys: A New Approach to Regulating Employment and Labour Markets. In: Transfer, 10 (2), S. 166-186.

\section{Rauchverbote in Deutschland: Ein thematischer Überblick}

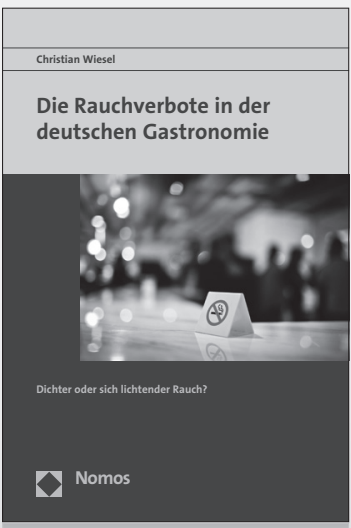

Nur wenige staatliche Maßnahmen haben zu einer derart lebhaften Debatte über Sinn und Unsinn geführt wie die Rauchverbote in der Gastronomie. In dieser Politikfeldanalyse werden die deutschen Nichtraucherschutzgesetze erstmals einer umfassenden Analyse unterzogen und ihre Entstehung beleuchtet. Mit Kompakt-Überblick über alle 16 Landesgesetze.

Die Rauchverbote in der deutschen Gastronomie

Dichter oder sich lichtender Rauch?

Von Christian Wiesel

2012, ca. 280 S., brosch., ca. 49,- €, ISBN 978-3-8329-6458-0

Erscheint ca. Juli 2012

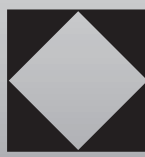

Nomos 